\title{
Erratum
}

\section{Efficient Catalytic Activity of Copper/Aluminum Hydrotalcite in Diaryl Ether Synthesis}

B. Sreedhar,* R. Arundhathi, M. Amarnath Reddy, M. Lakshmi Kantam Synthesis 2009, 483.

In the e-first version the name of the author M. Lakshmi Kantam was misspelled as M. Lakshmi Kanatm. It has been corrected for both the print and the current online versions. 